\title{
Tanner Scale Girl Version
}

National Cancer Institute

\section{Source}

National Cancer Institute. Tanner Scale Girl Version. NCI Thesaurus. Code C124704.

A standardized rating scale developed by Marshal and Tanner in 1969, which is a

classification system used to measure physical development in children, adolescents and adults. The girl version of the tanner scale assesses the different stages of physical

maturity based on breast development and the growth of pubic hair. This instrument has 5 stages. 